\title{
Variant Neo-Aortoiliac System Operation Using a Composite Conduit of Silver-Coated Dacron Graft and Autologous Femoral Veins: A Report of Two Cases
}

\author{
Petros K. Chatzigakis, Emmanouil M. Barmparessos, and Vasileios C. Katsikas \\ Vascular Surgery Department, General Hospital of Athens "Georgios Gennimatas”, Athens, Greece
}

\begin{abstract}
Aortic prosthesis infection is associated with significant morbidity and mortality rates. The introduction of the neo-aortoiliac system (NAIS) technique offers a new perspective, as a venous conduit is considered an optimal graft in aortic prosthesis infection because it is autogenous, durable, inexpensive and has low infection rates. Occasionally, the anatomical characteristics of available autologous venous conduits may limit the applicability of this technique. Herein, we present two cases in which a variant NAIS technique was used to confront an aortic graft infection. To expand the usefulness of the NAIS technique and avoid the use of prosthetic material in a potentially contaminated area as much as possible, we combined an autologous femoral vein with a short segment of a silver-coated Dacron graft. The combination of a silver-impregnated graft and the NAIS is a feasible bailout technique should the NAIS vein appears inadequate upon exploration.
\end{abstract}

Key Words: Prosthesis-related infections, Vascular grafting, Abdominal aorta, Femoral vein
Received March 17, 2021

Revised May 13, 2021

Accepted June 28, 2021

Published on July 9, 2021

Corresponding author: Petros K. Chatzigakis

Vascular Surgery Department, General Hospital of Athens "Georgios Gennimatas”, Av. Mesogeion 154, 15669, Athens, Greece

Tel: $30-2132033960$

Fax: 30-2132033470

E-mail: petros.chatzigakis@gmail.com https://orcid.org/0000-0002-9052-0389

Copyright $\odot 2021$ The Korean Society for Vascular Surgery

This is an Open Access article distributed under the terms of the Creative Commons Attribution Non-Commercial License (http://creativecommons.org/licenses/by-nc/4.0) which permits unrestricted non-commercial use, distribution, and reproduction in any medium, provided the original work is properly cited.

Cite this article; Vasc Specialist Int 2021. https://doi.org/10.5758/vsi.210022

\section{INTRODUCTION}

Aortic prosthesis infection poses a significant challenge for vascular surgeons. Its treatment requires explantation of the aortic graft, followed by restoration of blood perfusion. The neo-aortoiliac system (NAIS) operation, first described by Clagett et al. [1], has provided a new perspective on this lethal condition. In most cases, autologous veins can be used as arterial conduits. In a recent review, Pallister and Chung [2] showed how the femoral veins may be utilized for in situ reconstructions following meticulous planning and surgical techniques. However, there are sometimes unforeseen difficulties with the NAIS vein upon exploration, such as a large size mismatch, inadequate length, or fibrotic stenosis. Recently, we encountered two cases in which the superficial femoral vein was too short to perform the NAIS operation and thus treated them with a combination of silver-impregnated Dacron graft implantation and NAIS technique. This variant NAIS technique can preserve the benefits of the NAIS operation in the infected field and expand its applicability in various situations. The combination of a silver-impregnated graft and the NAIS is a feasible bailout technique should the NAIS vein appears inadequate upon exploration. Hence, we present two cases of aortic graft infection in which in situ aortic reconstruction was performed using this alternative technique.

Informed consent was obtained from the patients or the patients' next of kin prior to publishing their images and history. Therefore, approval by Institutional Review Board (IRB) was waived. 


\section{CASE}

\section{1) Case 1}

A 73-year-old male patient was admitted to an outlying general district hospital owing to an abscess in his right groin. Medical history-taking revealed open repair of an abdominal aortic aneurysm with an aortobifemoral bypass 3 years prior. Computed tomography angiography (CTA) revealed a pseudoaneurysm of the right common femoral artery at the site of the anastomosis, along with inflammatory perigraft tissue, extending to the retroperitoneal periaortic space and revealing a psoas abscess (Fig. 1A). After receiving intravenous antibiotics (ciprofloxacin and clindamycin) for 10 days, he was transferred to our hospital. We urgently proceeded to the operating room where under general anesthesia the graft was completely excised and sent for culture accompanied by the perigraft fluid. The aortic wall and surrounding tissues were debrided, and retroperitoneal irrigation was performed using an antibiotic solution (rifampicin). Subsequently, bilateral femoral veins were harvested in their total length by extending the dissections beyond the adductor magnus tendons. However, the lengths of both femoral veins were inadequate. Therefore, restoration of blood flow was accomplished using an in situ composite graft (Fig. 2A). A short segment of a bifurcated silver-coated Dacron graft was proximally sutured to the infrarenal aorta, and the distal graft limbs were anastomosed to the vein conduits, which were eventually linked to the femoral bifurcations (Fig. 3A). Owing to this configuration, the use of a prosthetic graft in the contaminated inguinal region was obviated, and the proximal anastomosis was managed with no significant mismatch owing to vein caliber discrepancy. After 5 days in the intensive care unit (ICU), the patient was transferred to the ward. Despite his lymphatic fistula in his right leg, which was treated conservatively with compression, the postoperative course was uneventful. He received intravenous meropenem and vancomycin during his hospital stay, followed by an oral regimen of ciprofloxacin and clindamycin for 6 weeks after his discharge on the 20th postoperative day in a good general condition. However, both the preoperative blood cultures and cultures of the excised graft and perigraft fluid yielded negative results.

\section{2) Case 2}

A 64-year-old male patient sought medical attention for recurrent episodes of fever and discomfort in his left thigh. He had a medical history of aortobifemoral bypass
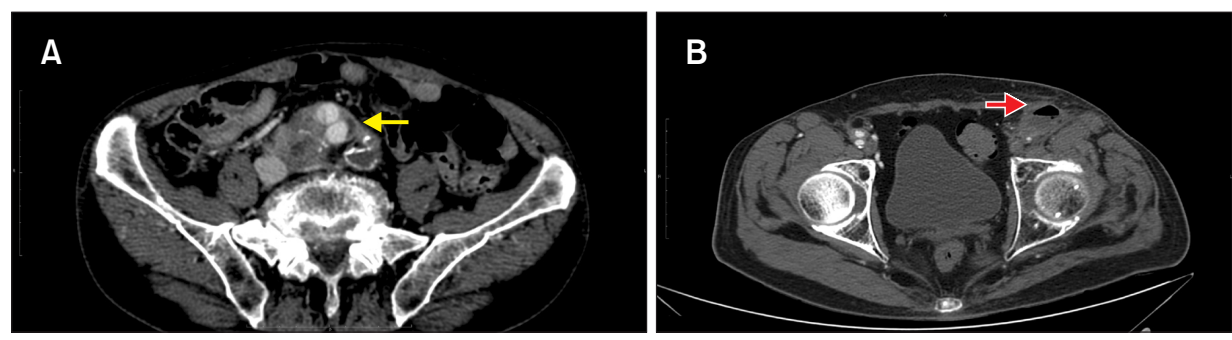

Fig. 1. Preoperative computed tomography scans. (A) Case 1. Yellow arrow indicates periaortic fluid and gas. (B) Case 2. Red arrow indicates inflammation tissue and gas encircling the femoral vessels.
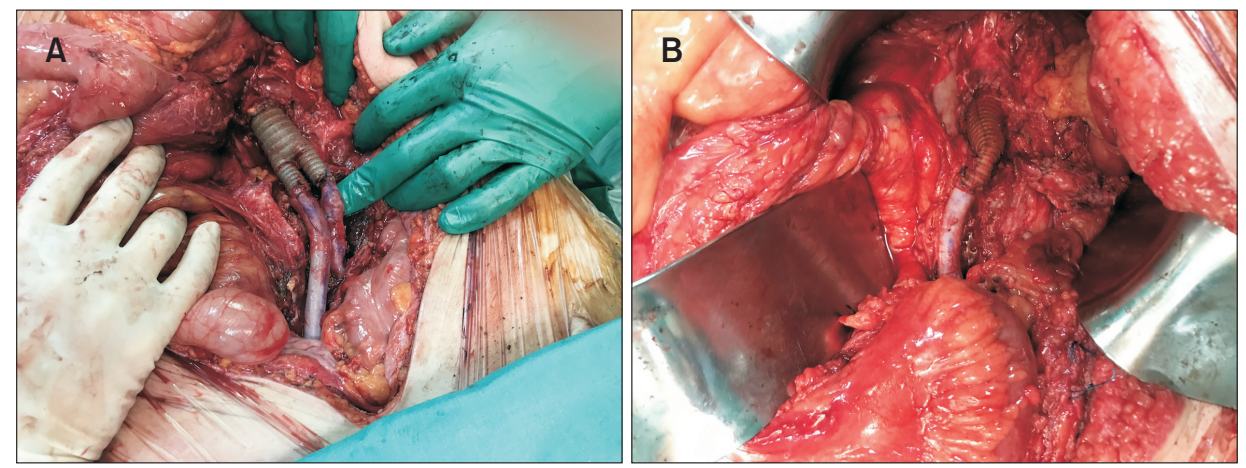

Fig. 2. Intraoperative images of the composite graft. (A) Case 1. A short segment of a bifurcated silver-coated Dacron graft was sutured to the infrarenal aorta proximally and to the autologous vein grafts distally. (B) Case 2. A short segment of a tubular silver-coated Dacron graft was sutured to the infrarenal aorta proximally and the autologous vein graft distally to the right side. The entire aortobifemoral graft was excised, including the thrombosed left limb, while debriding the surrounding infected tissue due to enteric fistula. 

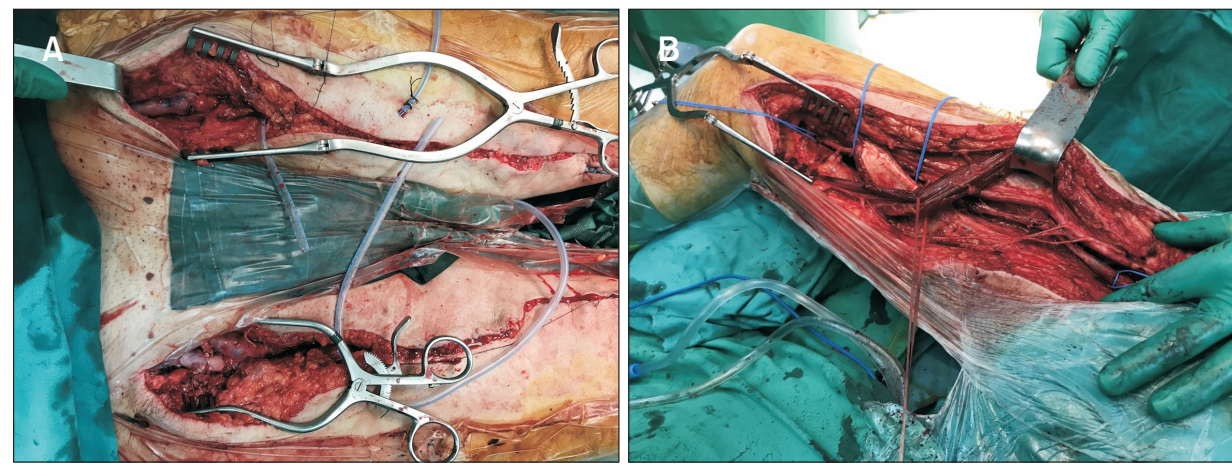

Fig. 3. Intraoperative images. (A) Case 1. Bilateral anastomoses of the autologous vein graft at the femoral bifurcation. (B) Case 2. Harvesting of the right femoral vein.

owing to aortoiliac occlusive disease 4 years prior. CTA revealed a retroperitoneal periaortic mass mainly on his left psoas extending downward, as far as the mid-thigh (Fig. 1B). Graft infection and abscess formation were suspected; however, blood cultures failed to exhibit any pathogenic microorganisms. There was also an inflammation near the cecum in proximity to the right limb of the graft, while the left limb of the graft was thrombosed. Despite the last finding, the patient did not complain of claudication. The patient was administered intravenous meropenem/vancomycin. Under general anesthesia, a long midline incision was made, and transperitoneal access revealed a fistula between the sigmoid colon and the left limb of the Dacron graft along with erosion of the cecum in proximity to the right limb. Thereafter, the entire graft was excised, and the aortic wall with the surrounding tissue was debrided and sent for culture. Retroperitoneal irrigation was performed using an antibiotic solution (rifampicin). Reperfusion was performed via in situ reconstruction of an aorto-right femoral bypass. For this reason, the right femoral vein was harvested in total length beyond the adductor hiatus (Fig. 3B). However, the length of the harvested femoral vein was considered inadequate despite preoperative planning. Hence, a composite conduit consisting of a short segment of a silver-coated Dacron graft and his right femoral vein was created (Fig. 2B). Considering the thrombosed left limb of the graft, sufficient collateral circulation of the left limb (ankle-brachial index of 0.6), and need to avoid the spread of inflammation and to shorten the operation time as much as possible because of his infirm condition, we proceeded without restoring the blood flow to his left limb. During the same procedure, sigmoidectomy and primary repair of the cecum lesion were performed. On the 5th postoperative day, he developed necrosis of the sigmoid colon, which was treated with Hartmann colectomy. Four days later, the patient experienced a rupture of the cecum repair site, which was confronted with right hemicolectomy. Cultures of the excised graft and perigraft tissue revealed the presence of multiple microorganisms possibly related to the aorto-en- teric fistula. Carbapenem-resistant Klebsiella pneumoniae, along with Escherichia coli, Proteus mirabilis, Enterococcus faecium, Bacteroides fragilis, Clostridium glycolicum, and Candida albicans, was isolated. Therefore, the antibiotic regimen was substituted with a combination of culturespecific antibiotics (i.e., daptomycin, metronidazole, ampicillin, anidulafungin, and ceftazidime/avibactam). Regretfully, after 38 days of ICU stay, the patient died of sepsis.

\section{DISCUSSION}

Aortic graft infection is a life-threatening complication that occurs in $1 \%$ to $2 \%$ of cases of conventional aortic surgery [3]. Its management is considered one of the most demanding situations in vascular surgery; it requires explantation of the infected graft and wide debridement followed by reperfusion. Various approaches have been presented as treatment plans; however, the most efficient treatment remains under discussion. The most widely accepted solution is extra-anatomic (axillobifemoral) bypass to reperfuse the viscera and the lower limbs, followed by excision of the infected prosthesis and proximal and distal ligations of the infrarenal aorta [4]. Several in-situ techniques have been described over the years. Each technique demonstrates its advantages over extra-anatomic bypass. The in situ technique involves the use of a wide variety of grafts, such as antibiotic-bonded grafts (e.g., rifampicinbonded Dacron grafts), silver-coated Dacron grafts, cryopreserved arterial homografts, and xenografts. The NAIS technique is a method of in situ reconstruction where the use of autologous veins offers significant advantages over the other techniques.

The goal of treatment is to improve the overall survival and limb salvage rates by offering a reperfusion technique with the highest long-term patency and the minimum rate of recurrent infection. Conversely, the operation should have the least impact on the patient's already poor condition. Despite significant progress in perioperative management, the mortality and morbidity rates remain high [5]. A 
meta-analysis by 0'Connor et al. [6] challenged the status of extra-anatomic bypass as the gold standard technique. They demonstrated several advantages of in situ graft replacement over extra-anatomic bypass, such as better graft patency, lower amputation and reinfection rates, and better overall survival.

The optimal conduit for use after aortic graft removal remains controversial. All types of conduits present limitations, and the success depends on many factors, mainly the patient's comorbidities and severity of infection. In a recent meta-analysis, Batt et al. [7] showed that although in situ reconstruction may be considered as a first-line treatment, the choice of the conduit should be based on variables, such as patient's age, aorto-enteric fistula, and virulence of the causative microorganisms. They showed that the reinfection rates were not significantly different among the available conduits. However, they concluded that silvercoated Dacron grafts yielded better outcomes in older patients, and autologous veins are probably the most suitable conduit to prevent reinfection. Silver-coated Dacron grafts have emerged as a reasonable option for the treatment of infected aortic grafts [8]. They are granted availability and relatively low rates of secondary procedures. Bisdas et al. [9] compared cryopreserved arterial homografts with silvercoated Dacron grafts for abdominal aortic infections and found no significant differences in the 2-year survival and limb salvage rates. Silver-coated grafts have several advantages: They do not contribute to antibiotic resistance and are quite effective in a wide range of bacteria, including methicillin-resistant Staphylococcus aureus species. In addition, they are available in different sizes and types; therefore, they have shown significant versatility, which allows them to be straightforward and simple to use [5].

Regarding NAIS operation, the theoretical advantages of this technique are the unique characteristics of the superficial femoral veins. The infection resistance and non-thrombogenic surface, along with their large caliber, make them an ideal conduit [10]. From a technical perspective, the NAIS operation is a demanding procedure, but still within the capabilities of a well-trained vascular surgeon. Ali et al. [11] showed that no "learning curve" is required. However, this approach has some important limitations. The NAIS technique is a time-consuming procedure that may last, on average, for 9 to 11 hours, adding a significant burden to an already fragile patient. In addition, the harvested veins occasionally have an inadequate length or caliber, and in the postoperative course, there is always a risk of deep vein thrombosis, compartment syndrome due to excessive limb edema, and lymphatic fistula following extensive incisions [2].

Herein, we present two cases of aortic graft infections.
Although the original strategy was to perform a NAIS operation, in view of the suboptimal femoral veins intraoperatively, we used a composite graft, attempting to combine the advantages of both the NAIS technique and silver-coated Dacron graft implantation. Autologous femoral veins are utilized as a durable conduit and less prone to reinfection in the contaminated inguinal area, while silver-impregnated graft implantation can readily obviate size discrepancies without complex modifications to the proximal aortic stump or vein graft.

In view of the inadequate length of the vein conduit, along with a mean diameter of 5 to $6 \mathrm{~mm}$ in both cases, and considering that the cause of the infection was confined distally to the aortic graft, mainly in the groins, we proceeded to use a composite graft rather than an entire silver-coated Dacron graft, which would have covered the regions all the way to the common femoral artery; this was because in the latter case, the reinfection risk would be theoretically much higher. After removing the infected prosthesis, we created a composite graft consisting of a short segment of the silver-coated Dacron graft and femoral venous limbs. In the first case, a bifurcated silver-coated Dacron graft was used, while in the second case, a tubular graft was used. Following the completion of the anastomoses in both cases, omentoplasty was performed to cover and guard the Dacron graft. By preserving the patency of the deep femoral veins, none of our patients exhibited deep vein thrombosis while we proceeded with respect to the lymphatic channel when performing a layered closure of the extensive longitudinal thigh incisions. In the first case, the graft remained patent without any sign of reinfection at 2 years of follow-up. In the second case, even if there was no sign of reinfection or malfunction of the composite graft, the colonic lesions and the inability to heal the intestinal anastomoses proved to be lethal.

Variations in the NAIS technique have been previously published. In one case, the preservation of a segment of the affected synthetic graft was combined with the use of both deep and superficial veins of the lower limbs; in another case, there was a unilateral inline replacement of the infected aortofemoral graft limb by the femoral vein without excision of the entire synthetic graft [1,12]. Herein, we present a variant NAIS technique in which a short segment of a silver-coated Dacron graft was combined with the harvested autologous femoral veins in a composite graft. To the best of our knowledge, this is the first report published in the English literature describing this composite graft. This approach creates a balance between the advantages and disadvantages of other types of in situ reconstruction. Our variation of the NAIS technique proved to be versatile and effective. It may be significantly useful in young patients, 
particularly in cases where the infection is confined distally to the pelvis or groins, and less virulent microbes are present.

In conclusion, for the treatment of aortic graft infection, a variant NAIS procedure using a composite conduit of silver-coated Dacron graft and autologous femoral veins may be a feasible technique in bailout situations where the NAIS technique alone cannot be completed because of the suboptimal femoral veins.

\section{CONFLICTS OF INTEREST}

The authors have nothing to disclose.

\section{ORCID}

\author{
Petros K. Chatzigakis \\ https://orcid.org/0000-0002-9052-0389 \\ Emmanouil M. Barmparessos \\ https://orcid.org/0000-0001-8984-0054 \\ Vasileios C. Katsikas \\ https://orcid.org/0000-0001-7441-6090
}

\section{AUTHOR CONTRIBUTIONS}

Concept and design: PKC, EMB. Analysis and interpretation: PKC, VCK. Data collection: EMB, VCK. Writing the article: PKC, EMB. Critical revision of the article: all authors. Final approval of the article: all authors. Statistical analysis: none. Obtained funding: none. Overall responsibility: PKC.
1) Clagett GP, Bowers BL, Lopez-Viego MA, Rossi MB, Valentine RJ, Myers $\mathrm{Sl}$, et al. Creation of a neo-aortoiliac system from lower extremity deep and superficial veins. Ann Surg 1993;218:239-248.

2) Pallister ZS, Chung J. Femoral vein reconstruction for aortic infections. Vasc Specialist Int 2021;37:4-13.

3) Svensson LG, Crawford ES, Hess KR, Coselli JS, Safi HJ. Experience with 1509 patients undergoing thoracoabdominal aortic operations. J Vasc Surg 1993;17:357-368.

4) Seeger JM, Pretus HA, Welborn MB, Ozaki CK, Flynn TC, Huber TS. Longterm outcome after treatment of aortic graft infection with staged extra-anatomic bypass grafting and aortic graft removal. J Vasc Surg 2000;32:451-459.

5) Chakfé N, Diener H, Lejay A, Assadian O, Berard X, Caillon J, et al. European
Society for Vascular Surgery (ESVS) 2020 clinical practice guidelines on the management of vascular graft and endograft infections. Eur J Vasc Endovasc Surg 2020;59:339-384.

6) O'Connor S, Andrew P, Batt M, Becquemin JP. A systematic review and meta-analysis of treatments for aortic graft infection. J Vasc Surg 2006;44:38-45.

7) Batt M, Feugier P, Camou F, Coffy A, Senneville E, Caillon J, et al. A metaanalysis of outcomes after in situ reconstructions for aortic graft infection. Angiology 2018;69:370-379.

8) Batt M, Jean-Baptiste E, O'Connor S, Bouillanne PJ, Haudebourg P, HassenKhodja R, et al. In-situ revascularisation for patients with aortic graft infection: a single centre experience with silver coated polyester grafts. Eur J Vasc Endovasc Surg 2008;36:182188.
9) Bisdas T, Wilhelmi M, Haverich A, Teebken OE. Cryopreserved arterial homografts vs silver-coated Dacron grafts for abdominal aortic infections with intraoperative evidence of microorganisms. J Vasc Surg 2011;53:12741281.

10) Chung J, Clagett GP. Neoaortoiliac System (NAIS) procedure for the treatment of the infected aortic graft. Semin Vasc Surg 2011;24:220-226.

11) Ali AT, Modrall JG, Hocking J, Valentine RJ, Spencer H, Eidt JF, et al. Long-term results of the treatment of aortic graft infection by in situ replacement with femoral popliteal vein grafts. J Vasc Surg 2009;50:30-39.

12) Simmons CD, Ali AT, Foteh K, Abate MR, Smeds MR, Spencer HJ, et al. Unilateral inline replacement of infected aortofemoral graft limb with femoral vein. J Vasc Surg 2017;65:1121-1129. 